\title{
Elaboração e implantação de protocolo de alta responsável para idosos com doenças crônicas hospitalizados e com necessidades alimentares especiais
}

\section{Development and implementation of responsible discharge care protocol for hospitalized elderly with chronic diseases and in need of special nutritional support}

\author{
Rubia Daniela Thieme' \\ Lauren Machado Pinto \\ Darla Silverio Macedo \\ Rosibeth del Carmen Muñoz Palm \\ Maria Eliana Madalozzo Schieferdecker ${ }^{4}$ \\ 1 Programa de Pós-graduação em Segurança \\ Alimentar e Nutricional. Universidade Federal do \\ Paraná. Curitiba, PR, Brasil. \\ 2 Programa de Pós-graduação em Medicina Interna \\ e Ciências da Saúde. Hospital de Clínicas da \\ Universidade Federal do Paraná. Curitiba, PR, Brasil. \\ ${ }^{3}$ Departamento de Terapia Ocupacional, \\ Universidade Federal do Paraná. Curitiba, PR, Brasil. \\ ${ }^{4}$ Departamento de Nutrição e do Programa \\ de Pós-graduação em Segurança Alimentar e \\ Nutricional. Universidade Federal do Paraná. \\ Curitiba, PR, Brasil. \\ Correspondência / Correspondence \\ Maria Eliana M. Schieferdecker \\ UFPR - Departamento de Nutrição \\ Av. Prefeito Lothário Meissner, 632 - Jd. Botânico \\ 80210-170 Curitiba, PR, Brasil \\ E-mail:meliana@ufpr.br
}

\section{Resumo}

Introdução: Idosos com doenças crônicas não transmissíveis (DCNT) hospitalizados frequentemente são frágeis e apresentam desnutrição e/ou necessidade de terapia nutricional (TN). Objetivo: Relatar a experiência de elaboração e implantação de protocolo referente à linha de cuidado para idosos hospitalizados devido a complicações decorrentes de DCNT com necessidade de TN e organizar as atividades preconizadas nesse protocolo de acordo com o planejamento da alta responsável e estruturação da linha de cuidado. Metodologia: Relato de experiência de elaboração e implantação de protocolo multiprofissional referente à linha de cuidado para idosos hospitalizados com DCTNs e necessidade de TN, entre agosto de 2011 e janeiro de 2012. Resultados: A participação do idoso e familiares/cuidadores foi primordial na elaboração do protocolo. A alta hospitalar responsável e a comunicação em saúde, por meio de visitas domiciliares, foram sistematizadas no planejamento e inseridas na linha de cuidado. Discussão: O protocolo sistematizado permitiu a continuidade de atenção à saúde preconizada na linha de cuidado por meio de estratégias de comunicação em saúde. A alta prevalência de desnutrição na admissão em atenção especializada sugere que a vigilância nutricional, o início precoce de $\mathrm{TN}$ e o acompanhamento do estado nutricional na Atenção Básica apresentam falhas. Quando necessário, o cuidado nutricional iniciado no hospital deve ter sua continuidade garantida no domicílio do indivíduo. Conclusão: A elaboração e implantação de protocolo de linha de cuidado, com planejamento da alta responsável, favoreceram a contrarreferência, sobretudo para continuidade de TN em domicílio.

Palavras-chave: Doença Crônica. Idoso. Alta Hospitalar. Comunicação em Saúde. Terapia Nutricional. Vigilância Nutricional. 


\section{Abstract}

Introduction: Elderly with chronic non-communicable diseases (NCD's) are often hospitalized with fragility and malnutrition and Nutrition Therapy (NT) may be needed. Objective: To report an experience of development and implementation of a care protocol for hospitalized elderly with NCDs complications and need of NT, and to organize protocol activities in accordance with responsible discharge and continued care planning. Methodology: Experience report of a transition and continued care protocol development and implementation for hospitalized elderly patients with NCDs, malnutrition, and need of NT, between August, 2011 and January, 2012. Results: Participation of elderly and their families/caregivers was central in designing the protocol. The responsible discharge and the communication in health, through home visits, were systematized on transition and continued care planning. Discussion: The systematized protocol allowed health care continuity across settings through communication strategies. The high prevalence of malnutrition in hospital settings suggests that nutritional surveillance, appropriate and early NT planning and monitoring of nutritional status in primary care are flawed. When indicated, nutritional support established in hospital settings must be continued in patient's home and NT must be ensured in primary care. Conclusion: Care protocol development and implementation, with transition and continued care planning and responsible discharge, supported health communication to continuity of NT at home.

Key words: Chronic Disease. Aged. Patient Discharge. Health Communication. Nutrition Therapy. Nutricional Surveillance.

\section{Introdução}

Na atualidade, as doenças crônicas não transmissíveis (DCNTs), consideradas um dos maiores problemas de saúde pública, são as principais causas de mortalidade no mundo, sobretudo em países em desenvolvimento, na população de menor renda e idosos. No Brasil, em 2007, as mortes em consequência de complicações das DCNTs atingiram $72 \%$ e, para o ano de 2015, estima-se que ainda será de 66\% dos óbitos. As doenças do aparelho circulatório, câncer, diabetes mellitus tipo 2 e doenças respiratórias crônicas são as relacionadas como as causas dos óbitos. ${ }^{1-3}$ 
A transição demográfica e a transição nutricional podem explicar parcialmente o modelo de transição da saúde. O envelhecimento da população contribui para o aumento da prevalência e incidência de DCNTs, mas suas principais causas são fatores de risco modificáveis, como a alimentação inadequada e o aumento da prevalência de sobrepeso e obesidade..$^{2,4,5}$

O padrão alimentar atual é constituído por alta ingestão de gorduras saturadas, sódio e açúcar refinado. Os alimentos considerados benéficos à saúde, ricos em fibras, vitaminas e minerais, são ingeridos em baixa quantidade. Manter a alimentação saudável pode auxiliar na prevenção e tratamento das DCNTs. ${ }^{3,6,7}$

A alimentação é, portanto, um dos condicionantes e determinantes da saúde. Segundo a Constituição Federal (CF) brasileira e a Lei Orgânica da Saúde (LOS), a qual regulamenta o Sistema Único de Saúde (SUS), a saúde é direito fundamental do ser humano, devendo o Estado, em conjunto com o próprio indivíduo, família, sociedade e empresas privadas, prover as condições para seu pleno exercício. Deste modo, o Direito Humano à Alimentação Adequada (DHAA) é também garantido na CF e considera a Segurança Alimentar e Nutricional (SAN) como meio para alcançá-lo. A SAN consiste na garantia do acesso regular, permanente e irrestrito a alimentos seguros em quantidade e qualidade seguras e adequadas, sem comprometer o acesso a outras necessidades essenciais, com base em práticas alimentares promotoras de saúde que respeitem a diversidade cultural e que sejam ambiental, cultural, econômica e socialmente sustentáveis, devendo o poder público adotar as políticas e ações que se façam necessárias para promover e garantir a SAN. ${ }^{8-11}$

A LOS determina a realização de ações de prevenção de doenças e agravos e promoção e recuperação da saúde. Neste aspecto, faz-se necessário também investir no diagnóstico precoce, com início imediato do tratamento adequado, a fim de evitar ou retardar as consequências nocivas para a saúde. ${ }^{12}$

As DCNTs e suas complicações demandam assistência continuada e intensiva em todos os pontos de atenção e cuidados à saúde, sobretudo aos idosos, devido a sua maior fragilidade, vulnerabilidade e dependência., ${ }^{4,5,13}$

Um dos princípios do SUS é a integralidade da assistência. Esta é conceituada como "conjunto articulado e contínuo das ações e serviços preventivos e curativos, individuais e coletivos, exigidos para cada caso, em todos os níveis de complexidade dos sistemas". A integralidade engloba ações de assistência e reabilitação, realizadas de forma articulada entre os pontos de atenção à saúde e com integração multiprofissional ${ }^{9,14,15}$ exigindo, portanto, estruturação de linha de cuidado. ${ }^{16,17}$ 
Indivíduos com DCNTs, devido às frequentes complicações que apresentam, podem necessitar de hospitalização, momento em que muitas vezes já estão desnutridos. A desnutrição é uma das doenças mais comuns no ambiente hospitalar. Estudos clássicos estimam que cerca de $50 \%$ dos indivíduos hospitalizados apresentam algum grau de desnutrição ${ }^{18,19}$ A desnutrição grave manifesta-se em aproximadamente $12 \%$ dos pacientes. ${ }^{18-20}$ Os idosos frequentemente possuem estado nutricional comprometido, decorrente sobretudo da doença de base, se neoplásica ou não, da diminuição da ingestão oral, capacidade digestiva reduzida, obstrução do trato gastrintestinal, doenças crônicas preexistentes, infecção e inflamação e de fatores socioeconômicos. ${ }^{21,22}$

As complicações decorrentes das DCNTs e a desnutrição podem determinar a necessidade de terapia nutricional (TN) durante a hospitalização, sobretudo para pacientes idosos. ${ }^{23-25}$ A TN consiste num conjunto de procedimentos terapêuticos aplicados como intervenção nutricional para manutenção ou recuperação do estado nutricional. As modalidades de TN são a parenteral, enteral e oral, realizadas nos diferentes pontos de atenção. ${ }^{26-28}$

Os casos em que o paciente deve dar continuidade à TN após a alta hospitalar por período de tempo prolongado são frequentes. A articulação de práticas de diferentes profissionais e pontos da rede de atenção à saúde favorece o engajamento do paciente e da família na TN em domicílio e pode ser estruturada a partir de uma linha de cuidado. ${ }^{29}$

A linha de cuidado estabelece os fluxos de integração dos pontos da Rede de Atenção à Saúde (RAS), conforme as necessidades do idoso. Nesse sentido, a estrutura da atenção especializada na lógica da RAS deve estar integrada aos outros pontos de atenção à saúde e ser eficiente para atenção dos casos de maior gravidade. A AB, como ponto de retorno do usuário após a alta da atenção especializada, deve estar apta para dar continuidade à assistência à saúde, ${ }^{13,16}$ destacando-se o tratamento de doenças e a reabilitação em domicílio. ${ }^{2}$

Os pontos de atenção à saúde na RAS estão em plano horizontal único e constituem rede progressiva e complementar de cuidados, devendo garantir a continuidade dos cuidados e promover a integração dos diferentes serviços. ${ }^{2}$ A AB tem função de atendimento das prioridades definidas localmente, enquanto que os serviços especializados, hospitais, centros de reabilitação, entre outros, têm o objetivo de atender a demandas de modo imediato e pontual, no uso de aparatos de maior tecnologia. Apesar dos aparentes objetivos distintos, a meta em comum é proporcionar atenção integral à saúde aos indivíduos. ${ }^{30,31}$

A fim de atender ao princípio de integralidade, é necessário que haja articulação e comunicação contínua entre os pontos de atenção à saúde e, dessa forma articulados, as demandas apresentadas pelo usuário podem ser atendidas. ${ }^{30} \mathrm{O}$ sistema de referência e 
contrarreferência do SUS é importante para que seus princípios sejam de fato efetivos, a fim de fortalecer a linha de cuidado. ${ }^{32,33} \mathrm{~A}$ intermediação segura entre a alta hospitalar e o retorno para o domicílio é realizada por meio de diferentes modalidades de atendimento das equipes de saúde, dentre as quais se destaca a visita domiciliar. ${ }^{34-36}$

A visita domiciliar integra a atenção em domicílio e refere-se a uma modalidade de intervenção, que pode ser promovida pelo hospital. Nessa modalidade, realiza-se contato pontual dos profissionais do hospital com o usuário, a fim de realizar coleta e/ou oferta de informações. Para este fim, ações de educação, capacitações de familiares e cuidadores e a articulação da RAS e outros setores de atenção são possíveis. ${ }^{37,38}$ Para tanto, a comunicação entre os pontos de atenção à saúde, na lógica da RAS, deve ser efetiva.

A comunicação entre pontos de atenção visa favorecer o acesso do usuário ao serviço, a busca de condições básicas de assistência, infraestrutura e recursos humanos qualificados. ${ }^{33}$ A continuação e manutenção do processo de educação do idoso e do cuidador em relação aos procedimentos prescritos e orientados no hospital e a adaptação dos mesmos à realidade do idoso e cuidador são funções da $\mathrm{AB}$ e podem ser favorecidas com a visita domiciliar. ${ }^{39}$

Para garantir a integralidade de assistência à saúde e o DHAA e consolidar os princípios do SUS, os modos de organização da atenção à saúde vêm sofrendo transformações com o objetivo de melhorar a articulação das práticas de diferentes profissionais e esferas da assistência e a adoção de modos mais eficientes de utilização dos escassos recursos disponíveis. ${ }^{29}$

A criação de protocolos que estabeleçam linha de cuidado de atenção em saúde é importante para aumentar a eficiência no cuidado às DCNTs e suas complicações. O protocolo, como descrito aqui, padroniza e normatiza uma linha de cuidado pensada para garantir a integralidade do cuidado ao idoso com DCNT, no período de internação, alta hospitalar e retorno ao domicílio.

Os protocolos devem contemplar ações multiprofissionais e intersetoriais, e favorecer a comunicação em saúde, por meio de procedimentos como visitas domiciliares, que objetivam a contrarreferência, a continuidade do cuidado e a integralidade da atenção. ${ }^{2}$ Os grupos populacionais mais vulneráveis, como os idosos, devem ser prioridade na execução das ações protocoladas.

O objetivo deste trabalho é relatar a experiência de elaboração e implantação de protocolo multiprofissional referente à linha de cuidado para idosos hospitalizados devido a complicações decorrentes de DCNTs e com necessidade de TN, e organizar as atividades preconizadas nesse protocolo de acordo com o planejamento da alta responsável e estruturação da linha de cuidado. 


\section{Metodologia}

Este estudo é um relato de experiência do desenvolvimento e implantação de protocolo multiprofissional referente à linha de cuidado de DCNT para idosos hospitalizados em hospital universitário de alta complexidade, localizado no município de Curitiba-PR, com as complicações decorrentes das DCNTs e com necessidade de TN. A linha de cuidado desse protocolo preconiza a implantação do Modelo de Atenção aos idosos com Doenças Crônicas na micropolítica, com objetivo de atuar como modelo para ações na macropolítica. ${ }^{2}$

A alta hospitalar responsável baseia-se na transferência do cuidado e é realizada por meio de orientação ao idoso e seu familiar/cuidador quanto à continuidade do tratamento, reforçando a autonomia do sujeito e proporcionando o autocuidado. O cuidado continuado deve ser realizado em todos os pontos de atenção, por meio da referência e contrarreferência, sendo a visita domiciliar um dos instrumentos.

O protocolo foi desenvolvido por residentes, preceptores e tutores do Programa de Residência Multiprofissional em Atenção Hospitalar, especificamente da Área de Concentração de Saúde do Adulto e Idoso (PSAI), em parceria e articulação com o Comitê de Humanização Institucional, de agosto de 2011 a janeiro de 2012. Os profissionais envolvidos nos anos de 2010 e 2011 foram: fisioterapeuta, nutricionista e terapeuta ocupacional; em 2011, ocorreu a inserção do psicólogo.

\section{Resultados}

De acordo com a percepção da equipe multiprofissional de atendimento hospitalar durante a rotina de assistência, os pacientes idosos admitidos nessa instituição hospitalar apresentavam múltiplas morbidades e maior número de complicações decorrentes de DCNTs além de, frequentemente, estado nutricional comprometido. Devido a esses fatores, geralmente permaneciam hospitalizados por tempo prolongado e eram expostos a múltiplos procedimentos (exames diagnósticos, TN, politerapia medicamentosa e outros). Sugere-se que a hospitalização, portanto, levou ao agravamento do quadro de fragilidade dos idosos. 
A Linha de Cuidado de Atenção ao Idoso estabelecida tem como base a identificação de riscos e integralidade do cuidado nos diferentes pontos de atenção à saúde. Uma vez identificado o risco, a prioridade centra-se em reduzir os impactos das DCNTs na funcionalidade do idoso. Para tanto, a articulação entre os pontos de atenção à saúde e a efetividade da assistência devem ser preconizados. ${ }^{40}$

No hospital, a Linha de Cuidado de Atenção ao Idoso estabelece o Projeto Terapêutico, resultado da discussão da equipe multiprofissional. O objetivo é avaliar ou reavaliar as condições de saúde e, se necessário, redefinir as linhas de intervenção terapêutica dos profissionais envolvidos no cuidado.

O Projeto Terapêutico faz parte do Protocolo de Alta Responsável, que é uma ferramenta utilizada na organização da linha de cuidado, estabelecendo fluxograma, procedimentos e orientações no período de internação, alta hospitalar e retorno ao domicílio, por meio de referência e contrarreferência. Estes são dispositivos administrativos utilizados para a comunicação entre pontos da RAS, com o objetivo de favorecer o acesso do usuário ao serviço e a continuidade dos cuidados. A visita domiciliar estabelecida como etapa de operacionalização do Protocolo de Alta Responsável tem como finalidade: encaminhamento junto à equipe de referência da $A B$; integração do idoso no seu ambiente social e familiar; reavaliação de orientações realizadas na alta hospitalar.

A equipe multiprofissional elencou critérios de inclusão para visita domiciliar com base na predisposição de riscos à integridade do idoso hospitalizado, de acordo com a profissão e área de conhecimentos (quadro 1), e elaborou fluxograma (figura 1) para melhor ilustrar a elegibilidade do paciente idoso e os encaminhamentos estabelecidos no protocolo.

Quadro 1. Critérios de inclusão para visita domiciliar como estratégia de contrarreferência no cuidado a idosos com DCNT desospitalizados no ano de 2011.

\begin{tabular}{|l|l|}
\hline Profissão & Critérios de inclusão para visita domiciliar \\
\hline Fisioterapia & Idoso com limitação funcional e/ou com doença crônica degenerativa. \\
\hline Nutrição & $\begin{array}{l}\text { Idoso em terapia nutricional e/ou com estado nutricional } \\
\text { comprometido. }\end{array}$ \\
\hline Psicologia & $\begin{array}{l}\text { Idoso em sofrimento psíquico e/ou que demande atendimento } \\
\text { psicoterápico. }\end{array}$ \\
\hline $\begin{array}{l}\text { Terapia } \\
\text { Ocupacional }\end{array}$ & $\begin{array}{l}\text { Idoso com comprometimento no desempenho nas atividades de vida } \\
\text { diária e necessidade de cuidador. }\end{array}$ \\
\hline
\end{tabular}




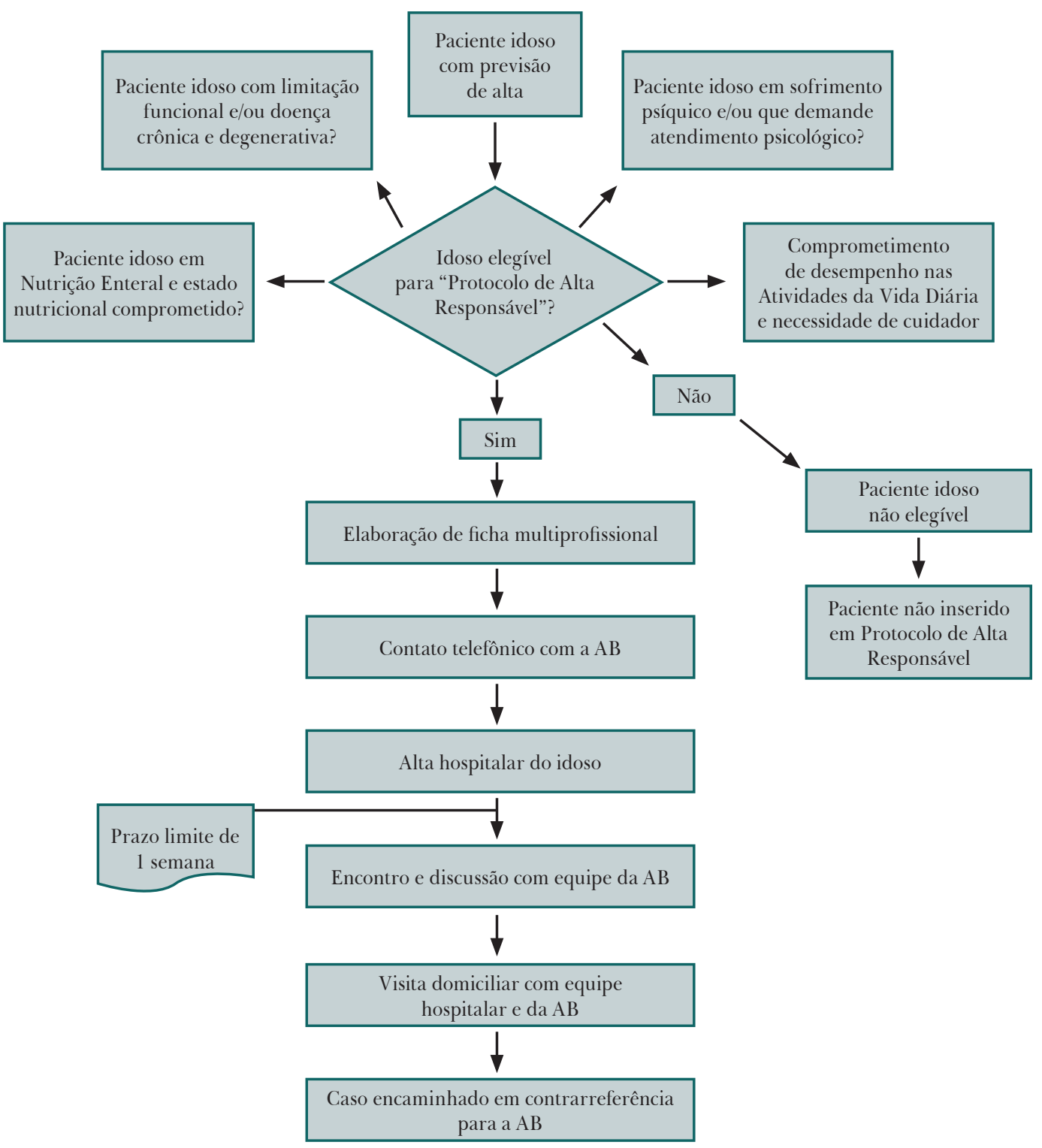

Figura 1. Fluxograma de atividades desenvolvidas em protocolo de alta responsável no ano de 2011. 
Para complementar a estratégia de contrarreferência, foi elaborado documento denominado "ficha de desospitalização", que foi preenchida em três vias pela equipe de assistência hospitalar. Esse documento é composto por dados referentes ao indivíduo, como endereço e vínculo com a $\mathrm{AB}$, ao histórico clínico e condutas dos membros da equipe multiprofissional relacionados ao período de permanência do idoso no hospital e perspectivas futuras quanto à continuidade do cuidado após o retorno ao domicílio. A ficha foi assinada pelos profissionais de saúde e pelo idoso ou cuidador.

Uma das vias da ficha de desospitalização foi entregue ao cuidador, o qual era responsável por entregá-la à Autoridade Sanitária da Unidade Básica de Saúde (UBS) antes da alta hospitalar, favorecendo o vínculo entre responsáveis pelo idoso e os profissionais da $\mathrm{AB}$. As outras duas vias destinaram-se, uma, à própria equipe hospitalar e outra, ao idoso.

A equipe hospitalar era responsável por realizar contato com a equipe da UBS de referência do idoso e agendar visita domiciliar em conjunto com representantes da UBS, a qual deveria ser realizada em até sete dias após a alta hospitalar. Anteriormente à visita em domicílio, aproximadamente um dia antes, um dos profissionais do hospital realizava contato telefônico com o cuidador e com representante da AB para confirmar a visita. A visita domiciliar foi realizada com a equipe multiprofissional hospitalar em conjunto com, no mínimo, um profissional da AB e, se possível e preferencialmente, com um agente comunitário de saúde. Essas ações favoreceram a estruturação e execução do planejamento. Para melhor compreensão do protocolo, foram elaborados objetivos, metas, ações, responsabilidades e avaliação das atividades desenvolvidas (quadro 2). 
Quadro 2. Planejamento da alta hospitalar e visitas domiciliares como estratégia de contrarreferência para idosos com complicações decorrentes de doenças crônicas não transmissíveis, estado nutricional comprometido e necessidade de terapia nutricional no ano de 2011.

\begin{tabular}{|c|c|c|c|c|}
\hline OBJETIVOS & METAS & AÇÕES & RESPONSÁVEL & AVALIAÇÃO \\
\hline $\begin{array}{l}\text { 1) Favorecer } \\
\text { a alta } \\
\text { hospitalar } \\
\text { responsável } \\
\text { de pacientes } \\
\text { idosos que } \\
\text { atenderam } \\
\text { aos critérios } \\
\text { de inclusão. }\end{array}$ & $\begin{array}{l}2.1) \\
\text { Comunicação } \\
\text { entre Atenção } \\
\text { Especializada } \\
\text { e AB. }\end{array}$ & $\begin{array}{l}\text { 1.1) Elaboração de } \\
\text { programa de alta } \\
\text { hospitalar. } \\
\text { 1.2) Preenchimento } \\
\text { da ficha de } \\
\text { desospitalização. } \\
\text { 1.3) Entrega da ficha } \\
\text { na UBS pelo cuidador. } \\
\text { 1.4) Orientação de } \\
\text { idoso e cuidador. } \\
\text { 1.5) Contato telefônico } \\
\text { com a UBS. } \\
\text { 2.1.1) Comunicação } \\
\text { inicial por meio } \\
\text { de ficha de } \\
\text { desospitalização. } \\
\text { 2.1.2) Visita da equipe } \\
\text { hospitalar à UBS. } \\
\text { 2.1.3) Reunião entre } \\
\text { equipe hospitalar e da } \\
\text { UBS. }\end{array}$ & $\begin{array}{l}\text { 2.2.1) Equipe } \\
\text { da Atenção } \\
\text { Especializada } \\
2.2 .2) \text { Equipe da } \\
\text { AB. } \\
\text { 2.2.3) Idoso } \\
\text { 2.2.4) Familiares } \\
\text { e/ou cuidadores. }\end{array}$ & 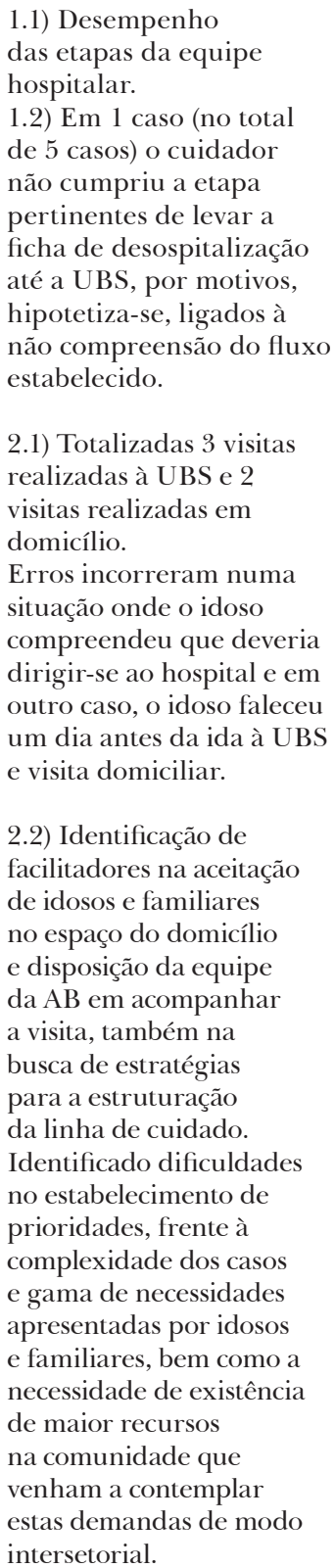 \\
\hline
\end{tabular}

UBS: Unidade Básica de Saúde; AB: Atenção Básica. 
Os resultados apontam que a estruturação e a execução da linha de cuidado envolvem as equipes de diferentes pontos de atenção à saúde, bem como o próprio idoso e seus familiares/ cuidadores, em todos os momentos desse processo complexo de ações de cuidado em saúde.

Os idosos hospitalizados incluídos no Protocolo de Alta Hospitalar Responsável apresentavam estado nutricional comprometido. A desnutrição é uma condição orgânica sistêmica. Portanto, indivíduos desnutridos podem apresentar comprometimentos na imunidade, na tolerância a exercícios e atividades cotidianas com maior risco de fadiga, alterações na percepção corporal, alterações na cognição e restrições na independência e autonomia.

A desnutrição era diagnosticada na avaliação nutricional completa realizada pelos nutricionistas do hospital. Após o diagnóstico, a TN era iniciada com o objetivo de manter ou recuperar o estado nutricional do indivíduo durante o período de hospitalização.

A equipe multiprofissional desenvolveu intervenções, propostas por cada área profissional, com foco nas especificidades do indivíduo, descritas no protocolo e baseadas nos critérios de elegibilidade. Medidas de orientação para organização da rotina, capacitação para proteção articular para conservação de energia e proteção articular em atividades cotidianas e capacitação de cuidadores são exemplos de estratégias desenvolvidas que contemplam maiores possibilidades de autonomia.

A contrarreferência, com visitas domiciliares como instrumento, permitiu à equipe multiprofissional da Atenção Especializada verificar se a realização das orientações fornecidas no hospital era possível no domicílio. No caso de impossibilidade, a comunicação entre as equipes de saúde dos diferentes pontos de atenção, em conjunto com idoso, família e cuidador, permitiu adequar as orientações às condições do domicílio e às possibilidades terapêuticas da AB. Após a alta hospitalar, a manutenção, o acompanhamento e evolução ou alteração da $\mathrm{TN}$ passavam a ser de responsabilidade da AB.

\section{Discussão}

O monitoramento da morbimortalidade em DCNT, componente essencial para a vigilância em saúde, é dever do SUS. ${ }^{2} \mathrm{O}$ fato de o idoso ser admitido no hospital com estado nutricional comprometido pode significar que os fatores de risco para DCNTs não foram controlados de modo eficaz, o diagnóstico clínico foi tardio, tratamento indicado não se realizou adequadamente, e houve falhas na vigilância e atenção clínica e nutricional na AB. Além disso, o idoso e familiares/cuidadores não estavam aptos a identificar sinais e sintomas da doença, de seu agravamento e de desnutrição. 
O desenvolvimento de ações focadas na atenção integral à saúde é um desafio na nova lógica de se pensar o SUS. O cuidado despersonalizado e fragmentado deve progressivamente evoluir ao conjunto de ações integradas nos diferentes pontos de atenção à saúde e para planos terapêuticos individualizados, que configuram linhas de cuidados. ${ }^{41} \mathrm{Na}$ especificidade das DCNTs, o fortalecimento do sistema de atenção à saúde inclui o fortalecimento da AB, com articulação com os-pontos de atenção da RAS. ${ }^{42}$ Esta sistemática responde ao objetivo de atenção às necessidades, demandas e desejos do cidadão usuário do SUS. ${ }^{41}$

Nesse pressuposto, a atenção sistematizada e embasada na realidade dos serviços e dos usuários configura necessidades e desafios postos ao SUS. ${ }^{41} \mathrm{Em}$ partes, pode-se dizer que o desenvolvimento do Protocolo de Alta Responsável, elucidado neste estudo, vem responder a um dos muitos desafios postos para o sucesso das linhas de cuidado.

O uso do protocolo, conforme apontado pelos resultados, consistiu na tentativa de abranger os múltiplos atores envolvidos no cuidado à saúde (idoso, familiar/cuidador, equipe hospitalar e equipe da UBS). A lógica de interlocução entre os serviços do hospital ao domicílio do idoso, passando pela $\mathrm{AB}$, foi um instrumento eficaz na identificação das reais demandas do idoso, bem como um mecanismo de empoderamento e responsabilização deste e de sua família no seu tratamento.

$\mathrm{Na} \mathrm{AB}$, foram identificadas limitações na estruturação de algumas equipes de saúde, que ainda não estavam inseridas na lógica da Estratégia Saúde da Família (ESF), bem como algumas UBS, que tinham amplo território de abrangência, limitando a ação integral ao idoso. Portanto, apontam-se desafios para a AB tanto na instrumentalização de suas equipes, na reflexão de novos fluxos de trabalho, quanto em investimento na criação de novos serviços, sobretudo UBS, adequadas à demanda populacional.

O planejamento estratégico é um mecanismo que pode ser eficaz para analisar a capacidade dos recursos empregados na produção dos resultados esperados. É frequentemente empregado na gestão dos serviços da área da saúde e tem como objetivo estudar as metas de uma intervenção, os meios empregados e os resultados produzidos. ${ }^{43}$ Assim, para o alcance de resultados conforme o planejado, sugere-se o uso dessa ferramenta para o estabelecimento de indicadores que permitam avaliar os resultados alcançados na operacionalização da linha de cuidados com idosos com DCNTs.

Refletindo a respeito da abordagem integral das DCNTs, constata-se que esta inclui a atuação em todos os pontos de atenção à saúde, no campo da macro e micropolítica. No campo da macropolítica, situam-se ações regulatórias, articulações intersetoriais e organização da rede de serviços; na micropolítica, atuação na linha de cuidado, vinculação e responsabilização do cuidador e produção de autonomia do usuário. ${ }^{44}$ Isso envolve, 
além de mecanismos políticos de regulação, ${ }^{41}$ medidas de suporte ao autogerenciamento (aconselhamento, educação e informação); ao sistema de saúde em equipes multidisciplinares; mecanismos de desenvolvimento de instrumentos eficazes à tomada de decisão (prática baseada em evidência) e medidas de comunicação em saúde (equipe-usuário e equipes de diferentes serviços). Portanto, o ponto central desse modelo é a produção de informações entre os serviços, a avaliação do usuário, a otimização do tratamento e o seguimento entre diferentes serviços..$^{2,42}$

Neste estudo, foi identificada a existência de algumas ações centradas na macro e micropolítica. O protocolo foi uma experiência na promoção da alta responsável e de estruturação de mecanismos estruturantes à linha de cuidado. Ao identificar a necessidade de se vincular o idoso com DCNT pós-alta hospitalar às equipes da $\mathrm{AB}$, reflete-se sobre a possibilidade da garantia e continuidade do cuidado na RAS. Os resultados apontam ações que garantem parte deste objetivo, reconhecendo-se que, além das limitações identificadas na vinculação de alguns idosos com a responsabilidade por seu cuidado, outros mecanismos na macro e micropolítica são necessários para este fim, como a pactuação intersetorial (sobretudo saúde e assistência social), desenvolvimento de tecnologias do cuidado que contemplem diferentes pontos de atenção à saúde, bem como novos mecanismos de processos de trabalho. ${ }^{41}$

Portanto, a equipe de saúde que assiste ao indivíduo com DCNT deve estar esclarecida quanto às consequências nocivas dessa condição, as quais podem variar em diferentes níveis de incapacidade, até a morte. O tratamento adequado, então, deve ser estipulado a fim de evitar ou retardar as consequências prejudiciais para a saúde ou, então, a atenção adequada às sequelas que não foi possível impedir, como a necessidade da TN. ${ }^{27,28,45}$

Os desafios da TN na RAS englobam desde a ausência de profissionais de saúde, sobretudo nutricionistas, em número suficiente ou aptos a prestar vigilância e atenção nutricional em TN, até a escassez de insumos e equipamentos para sua realização. Todos os membros da equipe multiprofissional devem ser capazes de verificar o comprometimento do estado nutricional de indivíduos na comunidade e no ambiente hospitalar, e serem treinados a encaminhar esses indivíduos ao atendimento de nutricionista, a fim de realizar o diagnóstico nutricional e o início da TN o mais precocemente possível, a fim de evitar danos mais graves.

O cuidado nutricional é capaz de atuar na promoção da saúde e na prevenção primária das DCNTs, bem como na prevenção secundária e terciária de complicações incapacitantes decorrentes dessas doenças. A necessidade de TN especializada, como por meio de sonda gástrica, é frequente quando as sequelas das complicações decorrentes das DCNTs impedem que o indivíduo mantenha alimentação via oral. Principalmente em casos mais graves, a atuação da equipe multiprofissional é importante no auxílio das adaptações físicas e 
psicológicas quanto à nova modalidade de alimentação, manejo e posicionamento do idoso e da sonda para evitar complicações, como a broncoaspiração, e aquelas que possam ocorrer em decorrência de alimentação inadequada, como as gastrintestinais.

Os custos referentes aos cuidados em DCNTs e suas complicações são elevados. Supõe-se que quanto maior a expectativa de vida, maiores são os gastos relativos à saúde agregados à presença de doenças crônicas e suas complicações. ${ }^{4,546}$ As modalidades de alimentação e nutrição especializadas e industrializadas também elevam os gastos, e podem ser onerosas para a família/responsáveis ou para o Estado.

Dessa forma, a implantação do protocolo, com realização de contrarreferência e visita domiciliar, possibilitou adequação da TN de acordo com os recursos disponíveis pela AB e no domicílio, substituindo-se fórmulas alimentares industrializadas de maior custo prescritas durante a hospitalização e orientadas na alta hospitalar por outra fornecida pela AB ou por alimentos. A transição de fórmulas ou de via de alimentação foi apoiada na presença das equipes dos dois pontos de atenção em saúde no território do paciente idoso, verificação da evolução clínica e nutricional após a alta hospitalar e garantia de continuidade do acompanhamento pela equipe da $\mathrm{AB}$. O vínculo entre o idoso e a $\mathrm{AB}$, estabelecido por meio de contrarreferência, é um dos mecanismos que podem garantir a continuidade do cuidado nutricional e clínico, o direito à saúde e o DHAA.

Assim, entre outros fatores, a ausência da efetividade do sistema de referência e contrarreferência leva ao retardo no processo de desospitalização. $\mathrm{Na} \mathrm{AB}$, a vigilância inadequada quanto à presença de DCNTs e suas complicações e à inadequação do estado nutricional leva ao aumento do número de admissões ou reinternações hospitalares. ${ }^{33,42,47}$

O desenvolvimento de ações sistematizadas na atenção à saúde do indivíduo com DCNT é um dos mecanismos eficazes na garantia da integralidade. O uso de protocolos pode ser útil na organização do processo de trabalho e na resolubilidade das ações de saúde. Apesar de limitado a atos e procedimentos preestabelecidos, quando o protocolo é estruturado de modo a atender às necessidades da população com DCNTs, pode ser instrumento que permite responder às reais demandas clínicas em diferentes situações. ${ }^{41}$

\section{Conclusões}

A elaboração e a implantação de Protocolo de Alta Responsável com estabelecimento de linha de cuidado é mecanismo viável de favorecimento de alta hospitalar responsável e de contrarreferência. O planejamento estipulado para o protocolo mostrou-se capaz de promover a articulação dos diferentes pontos de atenção em saúde e o cuidado integral ao indivíduo em TN. 
A RAS deve ser estruturada para atuar na prevenção dos agravos relacionados à alimentação e nutrição. Dessa forma, as ações voltadas aos indivíduos com necessidade de TN devem garantir o direito à saúde e o DHAA.

O relato desta experiência demonstrou a importância da equipe multidisciplinar na atuação no cuidado em necessidade de alimentação especial. A atenção interdisciplinar deve ser realizada de modo eficaz e sistematizado em todos os pontos de atenção à saúde.

O desafio no uso do protocolo descrito neste estudo está posto no desenvolvimento de indicadores objetivos e avaliação dos resultados, bem como na sistematização de outras ações que venham a favorecer a atenção integral ao sujeito com DCNT e necessidade de TN.

\section{Agradecimentos}

A Vânia Mari Salvi Andrzejevski, coordenadora do Programa de Residência Multiprofissional em Atenção Hospitalar, pelo apoio e orientações para a viabilidade deste projeto como protocolo para assistência aos pacientes, bem como aprendizagem dos residentes; e a Sirlei Corssetti Kleina, do Comitê de Humanização, pelos ensinamentos e apoio no desenvolvimento e implantação do protocolo, ambas do Hospital de Clínicas da Universidade Federal do Paraná (UFPR).

\section{Referências}

1. Schmidt MI, Duncan BB, Silva GA, Menezes AM, Monteiro CA, Barreto SM, et al. Doenças crônicas não transmissíveis no Brasil: carga e desafios atuais. Lancet 2011; 377(9781):1949-61.

2. Brasil. Ministério da Saúde. Plano de ações estratégicas para o enfrentamento da Doenças Crônicas não Transmissíveis (DCNT) no Brasil, 2011-2022. Brasília: Ministério da Saúde; 2011. 160 p.

3. World Health Organization. Global status report on noncommunicable diseases 2010. Geneva: WHO; 2011.

4. Lebrão ML. O envelhecimento no Brasil: aspectos da transição demográfica e epidemiológica. Saúde Coletiva 2007; 17(4):135-40.

5. Brasil. Ministério da Saúde. Atenção à saúde do idoso: aspectos conceituais. Brasília: Organização Pan-Americana de Saúde; 2012.

6. Brasil. Ministério da Saúde. VIGITEL Brasil 2009: vigilância de fatores de risco e proteção para doenças crônicas por inquérito telefônico. Brasília: Ministério da Saúde; 2010.

7. Szostak WB, Cybulska B, Kłosiewicz-Latoszek L, Szostak-Wegierek D. Primary prevention of cardiovascular disease and other chronic noncommunicable diseases in the centre of attention of the United Nations: special importance of a prudent diet. Kardiol Pol. 2013; 71(4):321-4. 
8. Brasil. Constituição da República Federativa do Brasil. Brasília: Senado Federal; 1988.

9. Brasil. Lei no 8.080, Lei Orgânica da Saúde. Diário Oficial da União19 set. 1990.

10. Brasil. Lei 11.346, Lei Orgânica de Segurança Alimentar e Nutricional. Diário Oficial da União [Internet] 15 set. 2006. Disponível em: http://www.abrandh.org.br/downloads/losanfinal15092006.pdf.

11. Burity V, Franceschini T, Valente F, Recine E, Leão M, Carvalho MF. Direito humano à alimentação adequada no contexto da segurança alimentar e nutricional. ação brasileira pela nutrição e direitos humanos (ABRANDH). Brasília: Ministério do Desenvolvimento Social e Combate à Fome; 2010.

12. Malta DC, Cezário AC, Moura L, Morais Neto OL, Silva Junior JB. A construção da vigilância e prevenção das doenças crônicas não transmissíveis no contexto do Sistema Único de Saúde. Epidemiol. Serv. Saúde. 2006; 15(3):47-65.

13. Brasil. Envelhecimento e saúde da pessoa idosa. Cadernos de atenção básica. Brasília: Ministério da Saúde; 2007.

14. Brasil. Conselho Nacional de Secretários de Saúde. Assistência de média e alta complexidade no SUS. Brasília: Conass; 2007.

15. Fratini JRG, Saupe R, Massarolli A. Referência e contra-referência: contribuição para a integralidade em saúde. Ciências Cuid Saúde 2008; 7(1):65-72.

16. Brasil. Portaria n 2.528 , de 19 de outubro de 2006. Aprova a Política Nacional de Saúde da Pessoa Idosa [Internet]. [acesso em 25 jun. 2012]. Disponível em: http://dtr2001.saude.gov.br/sas/PORTARIAS/ Port2006/GM/GM-2528.htm

17. Organização Panamericana de Saúde). Atenção à saúde do idoso: aspectos conceituais. Brasília: Ministério da Saúde; 2012.

18. Waitzberg DL, Caiaffa WT, Correia MITD. Hospital malnutrition: the Brazilian national survey (IBRANUTRI): a study of 4000 patients. Nutrition 2001; 17(7):573-80.

19. Correia MITD, Campos ACL. Prevalence of hospital malnutrition in Latin America: the multicenter ELAN study. Nutrition 2003; 19(10): 823-5.

20. Leandro-Merhi VA, Aquino JLB, Chagas JFS. Risco nutricional no período pré-operatório. Arq Bras Cir Dig 2009; 22(3):143-6.

21. Schiesser M, Müller S, Kirchhoff P, Breitenstein S, Schäfer M, Clavien PA. Assessment of a novel screening score for nutritional risk in predicting complications in gastro-intestinal surgery. Clin Nutr. 2008; 27(4):565-70.

22. Jensen GL, Mirtallo J, Compher C, Dhaliwal R, Forbes A, Grijalba RF, et al. Adult starvation and disease-related malnutrition: a proposal for etiology: based diagnosis in the clinical practice setting from the International Consensus Guidelines Comité. JPEN 2010; 34(2):156-159.

23. Volkert D, Berner YN, Berry E, Cederholm T, Coti Bertrand P, Milne A, et al. ESPEN guidelines on enteral nutrition: geriatrics. Clin Nutr 2006; 25(2):330-60.

24. Hutchinson E, Wilson N. Acute stroke, dysphagia and nutritional support. Br J Community Nurs. 2013; (Suppl):S26-9. 
25. Bazzan AJ, Newberg AB, Cho WC, Monti DA. Diet and nutrition in cancer survivorship and palliative care. Evid Based Complement Alternat Med. 2013; 2013:917647.

26. Brasil. Agência Nacional de Vigilância Sanitária. Resolução RDC no 63, de 06 de julho de 2000. Aprova o Regulamento Técnico para fixar os requisitos mínimos exigidos para a Terapia de Nutrição Enteral. Diário Oficial da União [Internet] 07 jul. 2000. Disponível em: http://portal.anvisa.gov.br/wps/wcm/ connect/61e1d380474597399f7bdf3fbc4c6735/RCD+N\%C2\%B0+63-2000.pdf?MOD=AJPERES

27. Candela CG, Cos Blanco A, García Luna PP, Cruz AP, Luengo Pérez LM, Iglesias Rosado C, et al. Complicaciones de la nutrición enteral domiciliaria. Resultados de um estúdio multicêntrico. Nutrición Hospitalaria 2003; 18:167-73.

28. Elia M, Stratton RJ. A cost-utility analysis in patients receiving enteral tube feeding at home and in nursing homes. Clinical Nutrition 2008; 27: 416-423.

29. Feuerwerker LCM, Merhy EE. A contribuição da atenção domiciliar para a configuração de redes substitutivas de saúde: desinstitucionalização e transformação de práticas. Rev. Panam. Salud Publica 2008; 24(3):180-8.

30. Organização das Nações Unidas para a educação, a ciência e a cultura. Estudos de contratualização de hospitais no âmbito do SUS. Rio de Janeiro: Fundação Getúlio Vargas; 2007. [acesso em 13 fev. 2013]. Disponível em: http://portal.saude.gov.br/portal/arquivos/pdf/Contratualizacao_1.pdf

31. Lopes WO, Saupe R, Massoroli A. Visita domiciliar: tecnologia para o cuidado, o ensino e a pesquisa. Cienc. Cuid. Saude 2008; 7(2):241-7.

32. Brasil. Ministério da Saúde. Atenção à saúde da pessoa idosa e ao envelhecimento. Área Técnica Saúde do Idoso. Série Pactos pela Saúde. Brasília: Ministério da Saúde; 2010.

33. Brasil. Ministério da Saúde. Manual instrutivo melhor em casa. Brasília: Ministério da Saúde; 2012. [Acesso em 25 jul. 2012]. Disponível em: http://189.28.128.100/dab/docs/geral/cartilha_melhor_ em_casa.pdf.

34. Brasil. Agência Nacional de Vigilância Sanitária. Portaria no 648, de 28 de março de 2006. Aprova a Política Nacional de Atenção Básica, estabelecendo a revisão de diretrizes e normas para a organização da Atenção Básica para o Programa Saúde da Família (PSF) e o Programa Agentes Comunitários de Saúde (PACS). Diário Oficial da União 29 mar. 2006. Disponível em: http://dtr2001.saude.gov.br/ sas/PORTARIAS/Port2006/GM/GM-648.htm

35. Chayamiti EMPC. Manual do serviço de assistência domiciliar. Ribeirão Preto: Secretaria Municipal de Saúde; 2007. (Manual Eletrônico) [acesso em: 12 nov. 2012]. Disponível em: http://www.ribeiraopreto. sp.gov.br/ssaude/programas/sad/manual-sad.pdf.

36. Teixeira, CP. Visita domiciliar: um instrumento de intervenção. Sociedade em Debate 2009;15(1):165-178.

37. Othero MB. Terapia Ocupacional na atenção extra-hospitalar oferecida pelo hospital. Cad. Ter. Ocu. UFSCar 2013; $20(2): 195-202$.

38. Brasil. Portaria no 963 de 27 de maio de 2013. Redefine a Atenção Domiciliar no âmbito do Sistema Único de Saúde (SUS). Diário Oficial da União 27 maio 2013. Disponível em: http://www.conass. org.br/conassinforma/anexo_963_maio_2013.pdf 
39. Brasil. Fundo Nacional de Saúde. Gestão Financeira do Sistema Único de Saúde: manual básico. $3^{a}$ ed. Brasília: Ministério da Saúde; 2003.

40. Veras RP, Caldas CP, Cordeiro HA, Motta LB, Lima KC. Desenvolvimento de uma linha de cuidados para o idoso: hierarquização baseada na capacidade funcional. Rev. Bras. Geriatr. Gerontol 2013; 16(2):385-392.

41. Werneck MAF, Faria HP, Campos KFC. Protocolo de cuidados à saúde e de organização do serviço. Belo Horizonte: Nescon/UFMG; Coopmed; 2009.

42. Brasil. Portaria no 2.527, de 27 de outubro de 2011. Redefine a Atenção Domiciliar no âmbito do Sistema Único de Saúde. Diário Oficial da União 28 out. 2011. Disponível em: http://bvsms.saude. gov.br/bvs/saudelegis/gm/2011/prt2527_27_10_2011.html

43. Contandriopoulos AP, Champagne F, Denis JL, Pineault R. A avaliação na área da saúde: conceitos e métodos. In: Hartz ZMA. Avaliação em saúde: dos modelos conceituais à prática na análise da implantação de programas [online]. Rio de Janeiro: Editora Fiocruz, 1997. p. 29-47.

44. Malta DC, Merhy EE. O percurso da linha do cuidado sob a perspectiva das doenças crônicas não transmissíveis. Interface - Comunic., Saúde, Educ. (Botucatu) 2010; 14:593-605.

45. Liley AJ, Manthorpe J. The impact of home enteral tube feeding in everyday life: a qualitative study. Health and Social Care in the Community 2003; 11(5):415-22.

46. Abegunde DO, Mathers CD, Adam T, Ortegon M, Strong K. The burden and costs of chronic diseases in low-income and middle-income countries. Lancet 2007; 370(9603):1929-38.

47. Goulart, FAA. Doenças crônicas não transmissíveis: estratégias de controle e desafios e para os Sistemas de Saúde. Brasília: Ministério da Saúde; 2011.

Recebido: 07/4/2014

Revisado: $27 / 5 / 2014$

Aprovado: 07/7/2014 